\title{
Myocardial Systolic Function Increases During Positive Pressure Lung Inflation
}

\author{
Michael F. Haney, Göran Johansson, Sören Häggmark, Björn Biber, \\ Michael F. Haney, MD, PhD, Göran Johansson, MS, Sören Häggmark, MS, and \\ Björn Biber, MD, PhD \\ Anesthesiology and Intensive Care Medicine, Umeå University Hospital, Umeå, Sweden
}

\begin{abstract}
Lung inflation with positive airway pressure may have rapid and dynamic effects on myocardial contractile function. We designed this study to assess the magnitude and time to onset of myocardial function changes during the initiation of single positive pressure lung inflation at clinically relevant inflation pressures. In 8 anesthetized $40-\mathrm{kg}$ pigs, left ventricular pressures and volumes were measured directly (conductance volumetry). A $15 \mathrm{~cm} \mathrm{H}_{2} \mathrm{O}$ airway pressure plateau with lung inflation (PPLI-15) was performed, and 2 single beats from that sequence, one from resting apnea at zero airway pressure and the second from the point when the
\end{abstract}

lungs were first maximally inflated, were selected for analysis. Systolic function variables for zero airway pressure and PPLI-15 were analyzed. Systolic elastance, derived from bilinear time-varying elastance curves, increased approximately 15\% during PPLI-15 from zero airway pressure. This agreed with other systolic function variables that identified an increase in left ventricular contractile function for the lung inflation beat. Serial measurements of myocardial function should be conducted with constant airway pressure and lung inflation conditions.

(Anesth Analg 2005;101:1269-74)
$\mathbf{P}$ ositive airway pressure and lung inflation can have distinct effects on heart loading conditions and performance that have been long recognized, although other direct and indirect effects on the heart have been suspected (1). In patients exposed to relatively low levels of positive airway pressure over longer periods, increased cardiac performance and sympathetic nervous system activation have been observed (2). Lung inflation with positive airway pressure may have dynamic effects on myocardial contractile status that have rapid onset, even during a single breath. Our hypothesis was that increases in myocardial contractile status occur very rapidly in response to positive airway pressure and lung inflation, and that this could be detected at the earliest point of full lung inflation during tidal breathing. The specific aim was to measure myocardial contractile function changes during onset and delivery of a single positive pressure lung inflation at clinically relevant inflation

Supported, in part, by grants from The Swedish Medical Research Council, The Swedish Heart-Lung Fund, and Umeå University, Sweden.

Accepted for publication June 21, 2005.

Address correspondence to Michael Haney, Anesthesiology and Intensive Care Medicine, Umeå University Hospital, 90185 Umeå, Sweden. Address e-mail to michael.haney@anestesi.umu.se.

DOI: 10.1213/01.ANE.0000181330.57600.FF pressures in an in vivo large animal model with an intact thorax.

\section{Methods}

With approval of the Ethical Research Committee of Umeå University, and in conformity with the Guide for the Care and Use of Laboratory Animals (National Academy of Sciences, 1996, USA), 8 Swedish Landrace pigs (mean weight $39.9 \mathrm{~kg}$ ) were used in this study. They were first premedicated using IM azaperone $2 \mathrm{mg} / \mathrm{kg}$ and ketamine $12 \mathrm{mg} / \mathrm{kg}$, and then anesthetized using IV pentobarbital $15 \mathrm{mg} / \mathrm{kg}$ and a pentobarbital infusion $15-20 \mathrm{mg} \cdot \mathrm{kg}^{-1} \cdot \mathrm{h}^{-1}$. No muscle relaxants were used. After a tracheotomy was performed, animals were ventilated with tidal volumes not exceeding $10 \mathrm{mg} / \mathrm{kg}$ (Servo 900B; SiemensElema, Stockholm, Sweden) to achieve normoxia and normocapnea (Oscar, Datex, Helsinki, Finland). Intravenous fluids were administered with the goal of maintaining normovolemia: Ringer's acetate solution $25 \mathrm{mg} / \mathrm{kg}$ during the first hour, then $10 \mathrm{~mL} \cdot \mathrm{kg}^{-1} \cdot \mathrm{h}^{-1}$ throughout the study period. Body temperature was measured and maintained between $38^{\circ} \mathrm{C}$ and $39^{\circ} \mathrm{C}$ using warmed IV fluids and a warming blanket. 
A multilumen central venous catheter (Arrow International, Reading, PA) and a pulmonary artery catheter (Optimetrix; Abbott, Abbott Park, IL) were placed via the external jugular vein system. An arterial catheter was placed with the tip in the descending aorta. A 7.5F balloon occlusion catheter (Vascular Technologies, Solna, Sweden) was placed into the inferior vena cava (IVC). Arterial pressure, central venous pressure (CVP), and pulmonary artery pressure were measured using a fluid-filled catheter system and transducers (Gabarith PMSET; Becton Dickinson, Franklin Lakes, NJ). A 7F left ventricular (LV) pigtail combination tip manometer and conductance catheter (CA-71083-PN; CD Leycom, Zoetermeer, Holland) were placed through an $8.5 \mathrm{~F}$ introducer in the carotid artery system into the LV using fluoroscopic guidance. Best conductance voltage signal and a stable catheter position at fluoroscopic examination were used to determine optimal conductance catheter position.

The conductance technique has been described in depth previously (3). LV volume was measured using the 12-electrode dual-field conductance catheter with $8 \mathrm{~mm}$ spacing between electrodes, and a signal conditioning-amplifier (Leycom Sigma 5DF; Cardiodynamics, Zoetermeer, Holland). Volume calibration included establishment of a flow reference ratio to the average of 3 thermodilution cardiac output measurements. Cardiac output was measured by thermodilution using the thermistor-tipped pulmonary artery catheter. Parallel conductance volume, by the hypertonic saline injection method (4), and blood conductivity were also measured. LV pressure and conductance data were recorded with a frequency of $250 \mathrm{~Hz}$ (PC Conduct; Cardiodynamics, Zoetermeer, Holland). All circulatory data were recorded and analyzed using a digital signal acquisition and analysis software package (Acqknowledge; Biopac Systems, Santa Barbara, CA).

The measuring sequences for the airway pressure increase were recorded beginning at zero airway pressure (ZEEP) with the endotracheal tube disconnected. The endotracheal tube was then connected to a pressure-cycled ventilator (Evita 4; Dräger, Lübeck, Germany) with $15 \mathrm{~cm} \mathrm{H}_{2} \mathrm{O}$ airway pressure plateau for $8 \mathrm{~s}$ (PPLI-15), where lung inflation occurred. The airway pressure was then released, and the measurement period ended. The animal was reconnected to the volume-cycled ventilator set to maintain normocapnea. In a separate measurement, a preload reduction sequence was recorded during ZEEP, brought about by a transient occlusion of the IVC by the balloon-tipped catheter.

From each airway pressure increase sequence, 2 single heart cycles were identified for analysis: one from the rest period at ZEEP before the positive airway pressure intervention and then a second heart cycle during PPLI-15. The heart cycle that was analyzed for PPLI-15 was systematically selected as the first heart beat, after the onset of PPLI-15, where CVP had increased and reached a stable level (Fig. 1). The time from onset of positive airway pressure $15 \mathrm{~cm}$ $\mathrm{H}_{2} \mathrm{O}$ until the start of the heart cycle measured during PPLI-15 was noted. Each heart cycle for ZEEP and PPLI-15 was analyzed for load and systolic function variables. End-diastole was determined either as the point 12 or $16 \mathrm{~ms}$ after the $\mathrm{R}$ wave onset, or based on milliseconds preceding the $\mathrm{dP} / \mathrm{dt}_{\max }>500 \mathrm{~mm}$ $\mathrm{Hg} \cdot \mathrm{s}^{-1}$. End-systole was determined for the single beats as maximal elastance (pressure/volume), or the point on the pressure-volume loop that was tangent to a line originating from the zero $\mathrm{x}$-intercept, with slope reported as Emax (5). All heart cycle times were measured and reported as heart rate (HR). Stroke work (SW) was calculated as the integral of LV pressure and volume during a single heart cycle. Time-varying elastance during each beat was analyzed for the ratio $(\alpha)$ of elastance slope during isovolumic contraction and ejection phases (6). End-systolic elastance (Ees) was determined as the linear regression slope for the endsystolic pressure-volume relation (ESPVR) over contiguous beats during the preload reduction sequence (7). For each animal, an Ees slope was measured for both the preload reduction sequence by transient IVC balloon occlusion at ZEEP and then separately from the preload reduction sequence that was induced by the PPLI-15 (8).

General circulatory variables for the 2 conditions (ZEEP and PPLI-15) were grouped for all 8 animals. Statistical inferences for differences within the paired measurements (ZEEP and PPLI-15) for all systolic function variables were made using a paired Student's $t$-test. Significant differences were determined if the $P$ value was $<0.05$.

\section{Results}

Differences in general circulatory and systolic function variables for paired beats at ZEEP and the PPLI-15 are shown in Table 1. The mean time from institution of positive airway pressure $15 \mathrm{~cm} \mathrm{H}_{2} \mathrm{O}$ to the beat that was analyzed for PPLI-15 was $1.5 \pm 0.1 \mathrm{~s}$ (SEM). CVP increased a small amount during PPLI-15, as demonstrated by a representative example in Figure 1. LV end-systolic pressure increased slightly from ZEEP to PPLI-15 whereas LV end-systolic volume decreased in all animals. In other words, the LV ejected to a lower end-systolic volume during PPLI-15, with no corresponding decrease in LV end-systolic pressure (LVESP). There were significant increases in the PPLI-15 measurement for both load-dependent systolic performance variables ejection fraction and SW, as well as load-indexed systolic function variables (SW/end-diastolic volume [EDV], Emax, and $\alpha$ ) as shown in Table 1. An example of representative paired 
Figure 1. This figure demonstrates a single representative measurement sequence with the single heart beats identified for zero expiratory airway pressure (ZEEP) (a), pressure plateau with lung inflation (PPLI-15) onset (b), and the single beat selected for measurement during PPLI-15 (c). Left ventricular pressure (LVP) and left ventricular volume (LVV) are plotted versus time and superimposed on each other in the upper left panel to demonstrate the temporal relation between the ZEEP and the PPLI-15 measurement points. The simultaneous central venous pressure (CVP) plot is shown in the lower left panel. A pressure-volume diagram of this same sequence (right panel) illustrates the progression of pressure and volume changes related to the institution of PPLI-15 (same events for $\mathrm{a}, \mathrm{b}$, and c). A separate preload reduction sequence by transient vena cava balloon occlusion is not shown here.
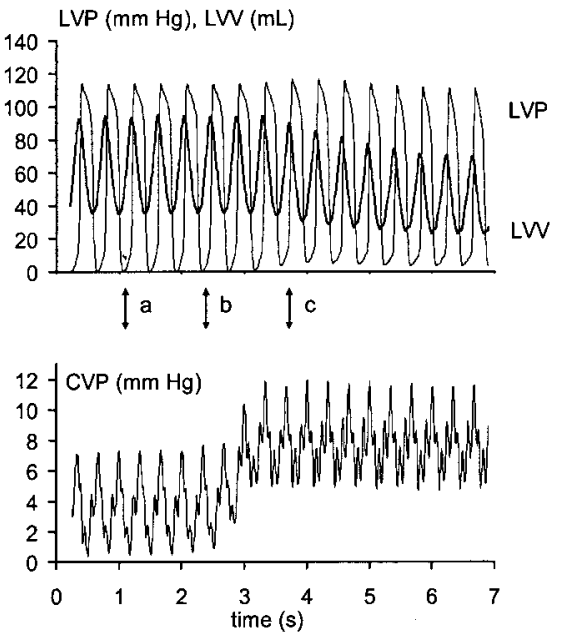

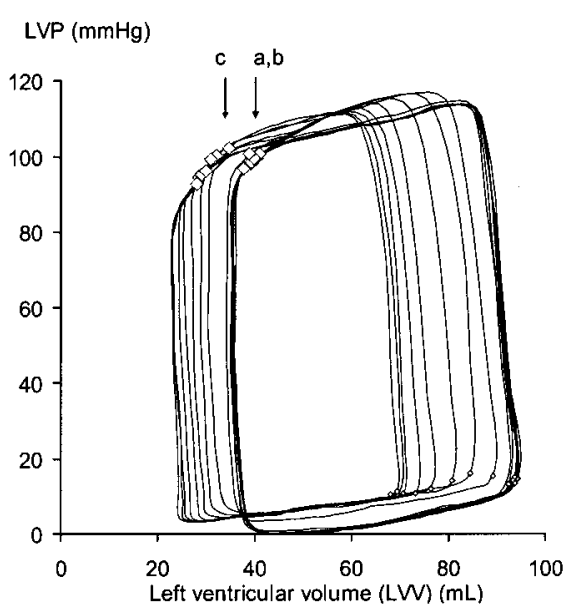

Table 1. Systolic Variables

\begin{tabular}{lcc}
\hline & ZEEP & PPLI-15 \\
\hline Single beat parameters & $99 \pm 18$ & $98 \pm 17$ \\
HR (bpm) & $6.4 \pm 1.7$ & $8.8 \pm 1.7^{*}$ \\
CVP (mm Hg) & $24 \pm 5.1$ & $27 \pm 4.5$ \\
MPAP (mm Hg) & $108 \pm 29$ & $103 \pm 27^{*}$ \\
EDV (mL) & $12.3 \pm 3.1$ & $15.5 \pm 4.2^{*}$ \\
EDP (mm Hg) & $66.2 \pm 26$ & $59.3 \pm 24^{*}$ \\
ESV (mL) & $112 \pm 14$ & $114 \pm 14^{*}$ \\
ESP (mm Hg) & $45 \pm 9$ & $49.3 \pm 11^{*}$ \\
EF $(\%)$ & $4253 \pm 789$ & $4456 \pm 761^{*}$ \\
SW (mm Hg/mL) & $42 \pm 13$ & $46.1 \pm 14^{*}$ \\
SW $/$ EDV (mm & & \\
$\quad$ Hg $\left.\cdot \mathrm{mL} \cdot \mathrm{mL}{ }^{-1}\right)$ & $2.0 \pm 0.9$ & $2.3 \pm 1.1^{*}$ \\
Emax (mm Hg/mL) & $0.47 \pm 0.20$ & $0.54 \pm 0.23^{*}$ \\
$\alpha$ & & \\
Derived from preload & & $1.34 \pm 0.4$ \\
$\quad$ reduction sequence & $1.20 \pm 0.5$ & \\
Ees (mm Hg/mL) & &
\end{tabular}

Values are mean $\pm \mathrm{SD} . n=8$

ZEEP = zero expiratory airway pressure; PPLI-15 $=15 \mathrm{~cm} \mathrm{H}_{2} \mathrm{O}$ airway pressure; $\mathrm{HR}=$ heart rate; $\mathrm{CVP}=$ central venous pressure; $\mathrm{MPAP}=$ mean pulmonary artery pressure; $\mathrm{EDV}=$ end-diastolic volume; $\mathrm{EDP}=$ enddiastolic pressure; ESV = end-systolic volume; ESP = end-systolic pressure; $\mathrm{EF}=$ ejection fraction; $\mathrm{SW}=$ stroke work; Emax = single beat end-systolic pressure-volume relation; $\alpha=$ single beat systolic time varying elastance; Ees $=$ end-systolic elastance

${ }^{*} P<0.05$, PPLI-15 value versus ZEEP using a paired Student's t-test.

observations with pressure-volume loops with timevarying elastance derivation is shown in Figure 2, along with an illustration of the derivation of the systolic function variable $\alpha$ in Figure 3. Ees slopes during PPLI-15 were not significantly different from the Ees measurements performed during ZEEP and transient vena cava occlusion.

\section{Discussion}

These results demonstrate that increases in LV contractile function measurements occur very rapidly during the course of single lung inflation in association with modest levels of positive pressure and inspiratory volume. This implies that myocardial function is dynamic during positive pressure breathing within single tidal respiratory cycles. This is the first study to observe a clear, rapid-onset myocardial function change in the pressure-volume plane with lung inflation pressures and volumes in this relatively low range.

When LV pressures increase during PPLI-15 (Table 1 and Fig. 1), this occurs despite a decrease in left ventricular end-diastolic volume (LVEDV, preload). It is not an increase in load, with unchanged myocardial contractile function, which causes this transient LVESP and systolic blood pressure increase. Instead, an increase in systolic function, occurring at a lower LVEDV (preload), produced this transient pressure increase. These findings are in contrast to the results of an earlier investigation (9) where a brief increase in preload was observed as a result of positive pressure lung inflation, which was suggested as the mechanism for a transient increase in stroke volume and systolic blood pressure, in the same setting. Differences in study material, conditions, and measurement methods for LV volumes are possible explanations for these apparently diverging results. The conclusions of that study: that increases in LVESP and systolic blood pressure occur because of increases in preload induced by an inspiratory "pump" mechanism, probably should not be considered a general or complete explanation for the transient inspiratory systolic pressure increase phenomena, in view of our results.

In the study design, there was an attempt to describe systolic function and loading from several aspects, including work (SW), the ESPVR, and time varying elastance $(\alpha)$. Also, a separate measurement for Ees, which used a preload reduction over consecutive beats, was used as a corroborating variable. Another form of analysis of ESPVR (10) for changes in 
Pressure / Volume, single heart cycle

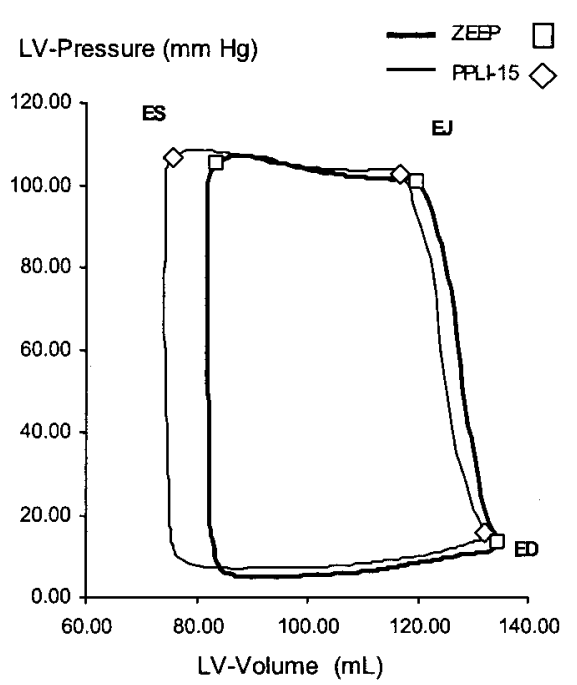

Pressure / Volume, single heart cycle

LV-Pressure $(\mathrm{mm} \mathrm{Hg})$

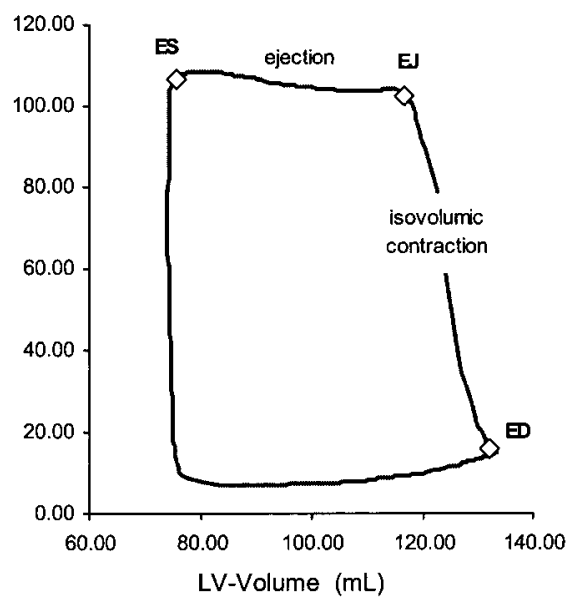

Time-varying Elastance during systole

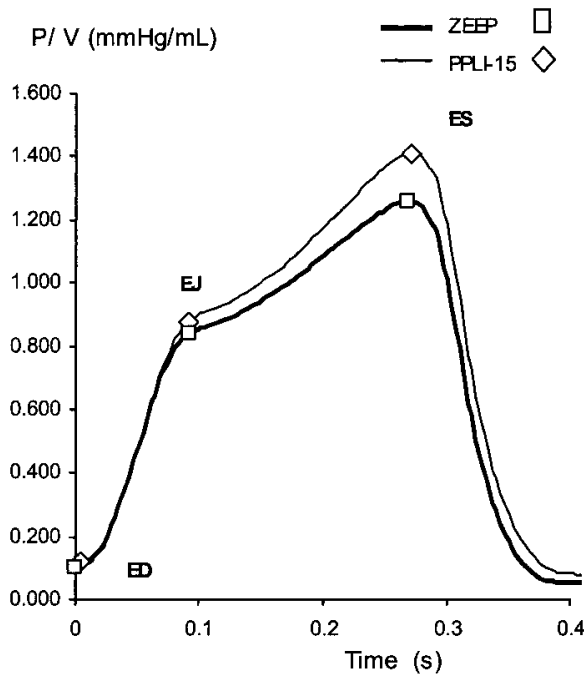

Time-varying Elastance during systole

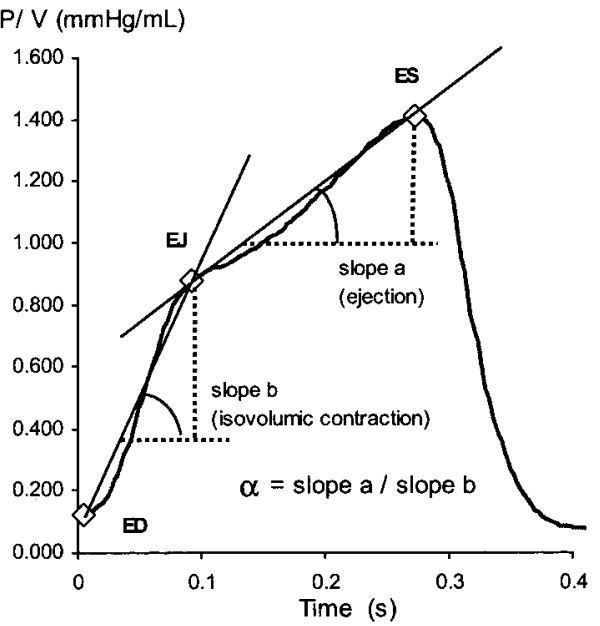

Figure 2. This figure shows a representative example of paired selected beats for zero expiratory airway pressure (ZEEP) and pressure plateau with lung inflation (PPLI-15) measurement with timevarying elastance (pressure/volume). ED $=$ end-diastole; $\mathrm{EJ}=$ start ejection; $\mathrm{ES}=$ end-systole.
Figure 3. This figure demonstrates the derivation of the systolic function variable $\alpha$ from a single representative heart cycle. The heart cycle-phase specific points are identified. ED = end-diastole; EJ = start ejection; ES = end-systole. The elastance slope for isovolemic contraction and ejection is used to calculate the ratio $\alpha$ (6). contractile status is to compare 2 beats from the same ventricle, which occur in the same end-systolic pressure range, and identify the beat where the ventricle ejects to a lower end-systolic volume as the beat with increased myocardial contractile status. This is more specific than Emax, given that the systolic events are compared at the same end-systolic load (pressure), or afterload. These ESPVR findings further support the finding of increased myocardial contractile function during PPLI-15.

In earlier clinical studies, increased ventricular performance, but not relation to ventricular load, has been described in a model of increased airway and intrathoracic pressures and experimental myocardial depression (11). Changes in ventricular volumes in response to positive airway pressure have also been described, as mentioned above (8). To assess myocardial function in response to these conditions, however, the relation of performance to load (and other conditions) must be measured accurately (12). Unless performance is indexed for the prevailing conditions, including load, then observations of change in performance do not provide direct information about the myocardial contractile status.

The time course of this response allows speculation that some form of autonomic nerve system activity is involved, although this study was not designed to identify a neurocirculatory mechanism. Increases in peripheral sympathetic nerve activity after initiation of positive pressure lung inflation in humans have been observed, although no myocardial function assessment has been performed during the immediate ensuing period after lung inflation $(13,14)$. One proposed mechanism is through stimulation of mechanical receptors in the lung and airway, which leads to activation of neurocirculatory reflexes (15). Regional 
blockade of lung afferent mechanoreceptor input during inflation has been shown to block increases in HR and arterial blood pressure in anesthetized patients, although more direct indices of myocardial function have not been measured (16).

Earlier physiological studies in animals have demonstrated that positive airway pressure and lung inflation over seconds or minutes appear to be able to initiate neurocirculatory reflexes that are modulated through lung mechanoreceptors and vagal efferent nerve signals that have been associated with bradycardia and even decreased ventricular contractile function (17-19). Other animal studies have suggested that lower positive airway inflation pressures can produce an early increase in HR with no clear effects on force of LV contraction (20) or a positive inotropic response (21). This body of evidence supports the possibility that a pulmonary afferent reflex and a neurocirculatory effector working in the LV could be involved, although further investigation is needed in this area.

Extracardiac pressure presumably increases to some extent during positive airway pressure lung inflation. The effect of this on transmural LV pressures can be measured in an invasive pericardial preparation, and there is evidence to suggest that even moderate or large continuous increases in extracardiac pressures do not alter the relationship of ventricular performance to loading conditions as quantified by, for example, preload recruitable SW (22). When large extracardiac pressures are present, transmural ventricular pressures need to be considered. Extracardiac pressure changes can be estimated, however, based on observations in changes in right atrial pressures (23) and are not large during PPLI-15. Systolic function results, including ESPVR and $\alpha$, are not affected by a small and constant difference in extracardiac pressure, making this very unlikely as a confounding factor.

The conductance volumetry method has been criticized regarding accuracy for absolute volumes, although the method is considered very reliable for precision or the identification of relative changes in volumes and direction of changes. There has been concern that lung inflation and positive pressure airway events can lead to a change in tissue volume signal conductivity surrounding the ventricle, potentially producing errors in measured volumes. There is evidence to suggest that no change occurs in parallel conductance during positive airway pressure lung inflation (24).

Also, with respect to parallel conductance, there is a theoretical concern that changes in right ventricular configuration could potentially cause a change in parallel conductance and ventricular volume signal. If a blood volume increase occurred in the right ventricle during positive pressure lung inflation and this was sensed by the conductance catheter as volume signal, then parallel conductance (and total conductancemeasured volume for the LV, but not true LV volume) would increase. The opposite was observed, however, with ventricular volume decreases during positive airway pressure. LV volume changes of this sort during positive pressure ventilation have been previously described (25).

The clinical significance of these modest variations in myocardial contractile function during the respiratory cycle may be recognition that they exist. When performing serial measurements of contractile status, the conditions must be standardized to the extent possible, and this includes lung inflation as a condition that can have effects on measured myocardial function. Therefore, analysis of myocardial contractile function should include attempts to standardize respiratory and lung inflation events during serial measurements. It is also possible that the myocardial response in an individual subject to lung inflation can change over time. The most certain method of eliminating potential myocardial effects of lung inflation from serial measures is to perform measurements at apnea with no positive airway pressure. Myocardial function measurements during respiratory activity can also be of interest, and longer respiratory pauses are not always practical for individual subjects. The data in this study indicate that serial measures of myocardial function need to have a consistent relation to the respiratory cycle.

In summary, a very rapid onset for increases in myocardial contractile function during a single positive airway pressure lung inflation was observed in this large animal model. The magnitude of the airway pressure and lung inflation was typical of that used for clinical support of ventilator-dependent subjects with healthy lungs. We conclude that the rapid variation in myocardial function in relation to positive pressure ventilation must be considered when measuring myocardial function, so that serial measures can be collected within the same context of cardiopulmonary interactions.

\section{References}

1. Van den Berg PCM, Jansen JRC, Pinsky MR. Effect of positive pressure on venous return in volume loaded cardiac surgical patients. J Appl Physiol 2002;92:1223-31.

2. Ziegler MG, Mills PJ, Loredo JS, et al. Effect of continuous positive airway pressure and placebo treatment on sympathetic nervous activity in patients with obstructive sleep apnea. Chest 2001;120:887-93.

3. Steendijk P, Van der Velde ET, Baan J. Left ventricular stroke volume by single and double excitation of conductance catheter in dogs. Am J Physiol 1993;264:H2198-207.

4. Steendijk P, Staal E, Jukema JW, Baan J. Hypertonic saline method accurately determines parallel conductance for dualfield conductance catheter. Am J Physiol Heart Circ Physiol 2001;281:H755-63. 
5. Suga H, Sagawa K, Shoukas AA. Load independence of the instantaneous pressure-volume ratio of the canine left ventricle and effects of epinephrine and heart rate on the ratio. Circ Res 1973;32:314.

6. Shoshido T. Hayashi K, Shigemi K, et al. Single-beat estimation of end-systolic elastance using bilinearly approximated timevarying elastance curves. Circulation 2000;102:1983-9.

7. Kass DA, Maughan WL, Guo ZM, et al. Comparative influence of load versus inotropic states on indexes of ventricular contractility: experimental and theoretical analysis based on pressure-volume relationships. Circulation 1987;76:1422-36.

8. Haney MF, Johansson G, Haggmark S, Biber B. Method of preload reduction during LVPVR analysis of systolic function: airway pressure elevation and vena cava occlusion. Anesthesiology 2002;97:436-46.

9. Vieillard-Baron A, Chergui K, Augarde R, et al. Cyclic changes in arterial pulse during respiratory support revisited by Doppler echocardiography. Am J Respir Crit Care Med 2003;168: 671-6.

10. Baan J, Van der Velde ET, Steendijk P. Ventricular pressurevolume relations in vivo. Eur Heart J 1992;13(SupplE):2-6.

11. Pinsky MR, Summer WR, Wise RA, et al. Augmentation of cardiac function by elevation of intrathoracic pressure. J Appl Physiol 1983;54:950-5.

12. Kass DA, Maughan WL. From 'Emax' to pressure-volume relations: a broader view. Circulation 1988;77:1203-12.

13. Macefield VG, Wallin BG. Modulation of muscle sympathetic activity during spontaneous and artificial ventilation and apnoea in humans. J Auton Nerv Syst 1995;53:137-47.

14. Sellgren J, Ponten J, Wallin BG. Characteristics of muscle nerve sympathetic activity during general anesthesia in humans. Acta Anaesthesiol Scand 1992;36:336-45.

15. Hamaya Y, Dohi S, Takenaka-Hamaya C. Localized airway anesthesia with lidocaine partially suppressed cardiovascular responses to lung inflation. Anesth Analg 2000;90:847-51.
16. Hamaya $Y$, Dohi S. Differences in cardiovascular response to airway stimulation at different sites and blockade of the responses by lidocaine. Anesthesiology 2000;93:95-103.

17. Kaufman MP, Iwamoto GA, Ashton JH, Cassidy SS. Responses to inflation of vagal afferents with endings in the lungs of dogs. Circ Res 1982;51:525-31.

18. Cassidy SS. Stimulus-response curves of the lung inflation cardio-depressor reflex. Resp Physiol 1984;57:259-68.

19. Ashton JH, Cassidy SS. Reflex depression of cardiovascular function during lung inflation. J Appl Physiol 1985;58:137-45.

20. Hainsworth R. Circulatory responses from lung inflation in anesthetized dogs. Am J Physiol 1974;226:247-55.

21. Greenwood PV, Hainsworth R, Karim F, et al. Reflex inotropic responses of the heart from lung inflation in anaesthetized dogs. Pflügers Arch 1980;386:199-205.

22. Haney MF, Johansson G, Haggmark S, Biber B. Analysis of left ventricular systolic function during elevated external cardiac pressures: an examination of measured transmural left ventricular pressure during pressure-volume analysis. Acta Anaesthesiol Scand 2001;45:868-74.

23. Smiseth $\mathrm{OA}$, Thompson $\mathrm{CR}$, Ling $\mathrm{H}$, et al. A potential clinical method for calculating transmural left ventricular filling pressure during positive end-expiratory pressure ventilation: an intraoperative study in humans. J Am Coll Cardiol 1996;27: 155-60.

24. Szwarc RS, Ball HA. Simultaneous LV and RV volumes by conductance catheter: effects of lung insufflation on parallel conductance. Am J Physiol 1998;275(2 Pt 2):H653-61.

25. Denault AY, Gorcsan J 3rd, Pinsky MR. Dynamic effects of positive-pressure ventilation on canine left ventricular pressurevolume relations. J Appl Physiol 2001;91:298-308. 